\title{
Fibroblast growth factor 2 enhances ATP release and extracellular purine metabolism in rat spinal astrocytes
}

\author{
Ryota Eguchi, Soichiro Yamaguchi, Ken-Ichi Otsuguro \\ Graduate School of Veterinary Medicine, Hokkaido University, Japan
}

\section{[Background]}

Extracellular adenosine (ADO) is known as an important neuromodulator in the central nervous system (CNS), and mediates neuroprotective effect under pathological conditions. In the CNS, extracellular ADO is mainly produced by ATP released from astrocytes. In the previous study, we showed that astrocytes release ATP via gap junction hemichannels (GJ $\mathrm{HCs}$ ) by extracellular $\mathrm{Ca}^{2+}$ reduction, one of the ischemic stimuli. Released ATP is metabolized to ADO via ecto-enzymes. It is also well known that astrocytes change their morphology and functions by pathological stimuli. However, it is unclear whether the astrocytic changes affect ATP release and purine metabolism. Therefore, in this study, we induced astrocytic changes by fibroblast growth factor 2 (FGF2) which increases under pathological conditions in the CNS, and investigated the ATP release and purine metabolism.

\section{[Methods]}

Cultured astrocytes isolated from rat spinal cord were treated with FGF2 for 2 days. Astrocytes were incubated with normal or $\mathrm{Ca}^{2+}$-free artificial cerebrospinal fluid (ACSF). Then extracellular purine levels were measured by highperformance liquid chromatography (HPLC) analysis and luciferin/luciferase method. Enzymatic activity for purine metabolism was measured by addition of ATP, AMP or ADO to ACSF and measurement of those metabolites with HPLC.

\section{[Results]}

In the normal astrocytes, $\mathrm{Ca}^{2+}$ reduction evoked ATP release, resulting in ADO increase by ATP degradation. FGF2 treatment potentiated the ATP release, and increased the expression and activity of GJ HCs. FGF2 also enhanced the metabolism of AMP to ADO and ADO to inosine. The expression levels of ecto-5'-nucleotidase (NT5E) and adenosine deaminase (ADA), AMP and ADO metabolizing enzymes respectively, were increased by FGF2. Extracellular ADA (ectoADA) activity was found in FGF2 treated astrocytes.

\section{[Conclusions]}

These results indicate that FGF2 potentiates ATP release and purine metabolism by increasing the expression and activity of GJ HCs, NT5E and ADA. Furthermore, it is suggested that FGF2 induces the ecto-ADA activity, although ADA has been considered to mainly act in cytosol in astrocytes. Astrocytes functionally changed by FGF2 may take an important role to control extracellular purine levels under pathological conditions. 